\title{
Estado del arte, optimización del modelo RLC y retos de fabricación de interconectores para alta frecuencia con base en nanotubos de carbono*
}

\section{Irving Larruz-Castillo ${ }^{1}$ \\ Aníbal Pacheco-Sánchez ${ }^{2}$ \\ Donato Valdez-Peréz ${ }^{1}$}

\author{
${ }^{1}$ Instituto Politécnico Nacional \\ Escuela Superior de Ingeniería Mecánica y Eléctrica Unidad \\ Zacatenco, Sección de Estudios de Posgrado e Investigación, \\ Edificio Z4 3er piso \\ 07738, Ciudad de México \\ MÉXICO \\ 2Universitat Autonoma de Barcelona \\ Department d'Enginyeria Electronica \\ 08193, Bellaterra (Cerdanyola del Vallès) \\ ESPAÑA
}

correos electrónicos (emails): ilarruzc1700@alumno.ipn.mx

anibaluriel.pacheco@uab.cat

dvaldez@ipn.mx

Recibido 31-01-2020, aceptado 29-05-2020.

\section{Resumen}

En el siguiente documento se hace una revisión bibliográfica sobre las propiedades eléctricas de los nanotubos de carbono $(\mathrm{CNT})$ en altas frecuencias y como han sido implementados como interconectores en diferentes dispositivos. Se muestran los resultados de dichas implementaciones y se analizan para su interpretación. De la bibliografía revisada, se selecciona un modelo RLC de interconectores con base en CNT y se hace un estudio del mismo. Se obtiene una función para la impedancia del dispositivo y utilizando los valores teóricos sugeridos para el modelo RLC, se extiende su análisis en altas frecuencias $(<100 \mathrm{GHz})$. A partir de la representación matricial

*Este artículo es la versión extendida de los mejores trabajos del Congreso Nacional de Ingeniería Electromecánica y de Sistemas 2019 tipo $\mathrm{ABCD}$ de los componentes del circuito equivalente, se calculan las ecuaciones de los parámetros de dispersión $(S)$ para este dispositivo. A partir de esta ecuación característica se realiza una optimización de los componentes del circuito RLC hacia una correcta descripción de los datos experimentales del dispositivo. Por último, se discuten los retos en la fabricación de interconectores con base en CNT.

Palabras clave: ecuación de impedancia, modelo compacto, parámetros $\mathrm{S}$, parámetros $\mathrm{ABCD}$, estado del arte.

\section{Abstract \\ (State-of-the-art RLC Model Optimization and Fabrication Challenges of High-Frequency Carbon Nanotube-Based Interconnectors)}

A literature review about the carbon nanotubes (CNTs) highfrequency electrical properties and their applications is exhibited on the following paper. The results of these implementations are shown and analyzed. A CNT-based RLC model for interconnectors is selected and a brief analysis of it is performed on detail. The device impedance function is obtained and by using the suggested values of the CNT-interconnects an analysis at high frequencies $(<100 \mathrm{GHz})$ is presented. The device scattering parameters $(S)$ equations are calculated from the $\mathrm{ABCD}$ matrix representation of equivalent circuit components. An optimization of such characteristic equation using the RLC circuit components towards the description of the experimental device data is performed. Lastly, the challenges of the CNTs based interconnectors fabrication used on different devices are discussed.

Index terms: $\mathrm{ABCD}$-parameters, compact-model, impedance equation, literature review, S-parameters, literature review.

\section{Introducción}

Gran número de importantes compañías han perseguido el diseño eficiente de los transistores, los cuales ofrezcan velocidades de respuesta elevados y a su vez sean lo más com- 
pactos posibles. La miniaturización de los transistores ha permitido mejoras en los circuitos integrados (CI) tanto en el desempeño, potencia, reducción de costos de fabricación y mayor densidad de transistores embebidos que pueden optimizar la funcionalidad de los CI [1].

Además de perseguir las características descritas anteriormente en un transistor, la industria de los semiconductores busca propiedades específicas en interconectores las cuales se ven afectadas por el escalamiento en dimensión [2], [3]. Cuando el tamaño del dispositivo disminuye la resistencia producida por los interconectores aumenta, sin mencionar que a mayor densidad de interconectores la capacitancia de acoplamiento entre ellos y los dispositivos activos aumenta [1].

El propósito fundamental de un interconector es proporcionar una comunicación entre dos regiones o bloques dentro de un CI por medio de señales, las cuales pueden ser señales de alta frecuencia, de potencia, entre otras. El verdadero desafío al diseñar un interconector será implementar una estructura adecuada para el tipo de señal que será transmitida de un bloque a otro, para ello se hacen uso de diferentes métricas como lo son: el retraso de la señal, el ancho de banda necesario, la cantidad de potencia que se transmite, inclusive el área que se utilizará dentro del CI. Estas métricas mencionadas estarán ligadas a las propiedades físicas del material utilizado como interconector, es decir, su resistencia por longitud, la capacitancia e inductancia producida por la estructura del interconector, etcétera.

Para describir de manera general la tecnología actual usada en un CI, la Fig. 1 [1] muestra un tipo de interconector con base en nanotubos de carbono (CNT, por sus siglas en inglés). La tecnología actual de fabricación implementa vías transversales de silicio (TSV por Through-Silicon Vias) en 3 dimensiones (3D) para conectar diferentes capas de transistores distribuidos en todo el volumen en los CI. En otras palabras, una TSV es un interconector vertical que "cruza o atraviesa" el silicio para conectar a dos segmentos diferentes de un CI.

En los últimos años, la implementación de los CNT ha constituido una de las nuevas alternativas para la fabricación de dispositivos, por ejemplo, diodos los cuales mostraron un mejor desempeño en altas frecuencias que los tradicionales de silicio [4]. En interconectores, se ha encontrado que ciertos arreglos de CNT presentan menor retraso en la señal en comparación con interconectores de la misma longitud fabricados con cobre [5], además esta mejora en el retraso de la señal puede mantenerse en altas frecuencias y ha sido documentada en trabajos donde se implementan interconectores con base de CNT en CI de osciladores [6], [7].

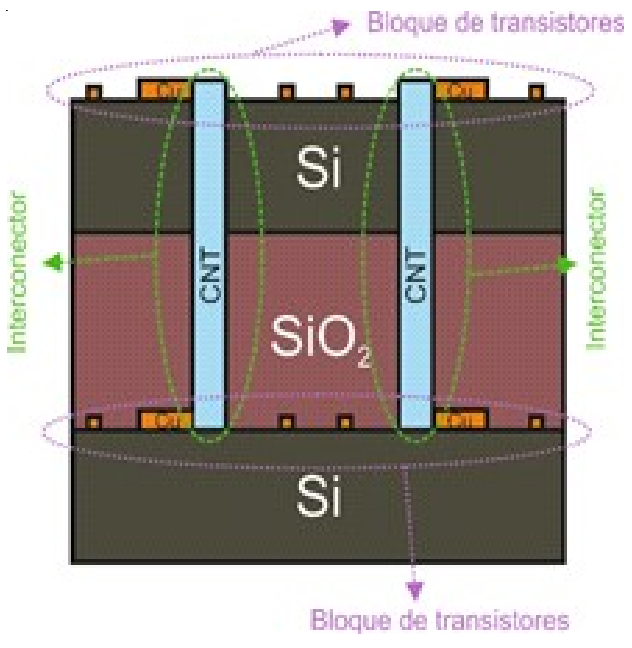

Fig. 1. Vista transversal de un $\mathrm{CI}$ (por ejemplo, un microprocesador) compuesto por 2 bloques de transistores los cuales se comunican mediante 2 interconectores a base de CNT que se implementan en TSV. Imagen adecuada de [1].

En contraste con los interconectores de cobre, los interconectores con base de CNT ofrecen, a la misma escala de implementación, ventajas en resistividad (mucho menor debido a su capacidad de transporte cuasi-balístico), mayor tolerancia electromagnética y mayor conductividad térmica [5], [8].

Algunos métodos de transmisión de datos hacen uso de anchos de banda en el orden de los GHz [9], aunado a esto, actualmente hay pocos estudios sobre modelos de interconectores con base en CNT que describan su comportamiento en altas frecuencias [10], [11]. Es por ello que en este artículo se retomarán, en la sección 2 algunas de las características en los CNT reportadas en altas frecuencias, así como posibles aplicaciones de los CNT. En la sección 3 se retoma un modelo RLC propuesto, así como valores planteados para cada elemento. Después, en la sección 4 se describe, mediante la simulación del modelo presentado en la sección 3, el comportamiento de un interconector con base en CNT de altas frecuencias $(<100 \mathrm{GHz})$ así como la optimización del mismo para la obtención de valores similares a los valores medidos. Por último, la sección 5 engloba de manera general algunos de los retos a enfrentar cuando se fabrican dispositivos que implementan CNT.

\section{Estado del arte de los interconectores en alta frecuencia a base de CNT}

El transporte de electrones en CNT metálicos fue caracterizado en [12], tanto en ambiente dinámico como en estático. Se reveló, que en los CNT metálicos la conductancia en DC (Co- 
rriente Directa o Direct Current) es aproximadamente igual a la conductancia en AC (Corriente Alterna o Alternating Current), al menos en un $50 \%$ del rango estudiado (DC a 10 $\mathrm{GHz}$ ). Los 2 dispositivos utilizados (A y B) para llegar a la conclusión de éste estudio consistían de dos contactos metálicos conectados por medio de un CNT de pared simple (SWCNT) de diferentes longitudes para cada uno ( $1 \mu \mathrm{m}$ para el dispositivo A y $25 \mu \mathrm{m}$ para el dispositivo B). En la Fig. 2 se muestran las conductancias obtenidas en ambos dispositivos [12]. El propósito fundamental de este estudio fue demostrar que a través de $1 \mathrm{CNT}$ se pueden transmitir señales, así como estimar el comportamiento de la impedancia al aumentar la frecuencia.

Un factor importante en la aplicación en altas frecuencias es la inductancia cinética de los CNT, debido a que esta propiedad aminora el efecto pelicular (en comparación con interconectores a base de $\mathrm{Cu}$ ) en frecuencias contiguas a las decenas de gigahertz, tal como se menciona en [13]. La inductancia cinética se puede definir como el exceso de energía cinética en los electrones del CNT. Por otro lado, el efecto pelicular es aquel que se produce cuándo corrientes alternas de altas frecuencias transitan por un conductor, dichas corrientes tienden a circular únicamente por la superficie de los mismos, incrementando esta tendencia en altas frecuencias, produciendo que la resistencia en AC aumente y difiera de la resistencia en DC. Para verificar esta propiedad de la inductancia cinética y tomando como base el dispositivo fabricado (guía de onda coplanar) de estructura G-S-G (estruc-

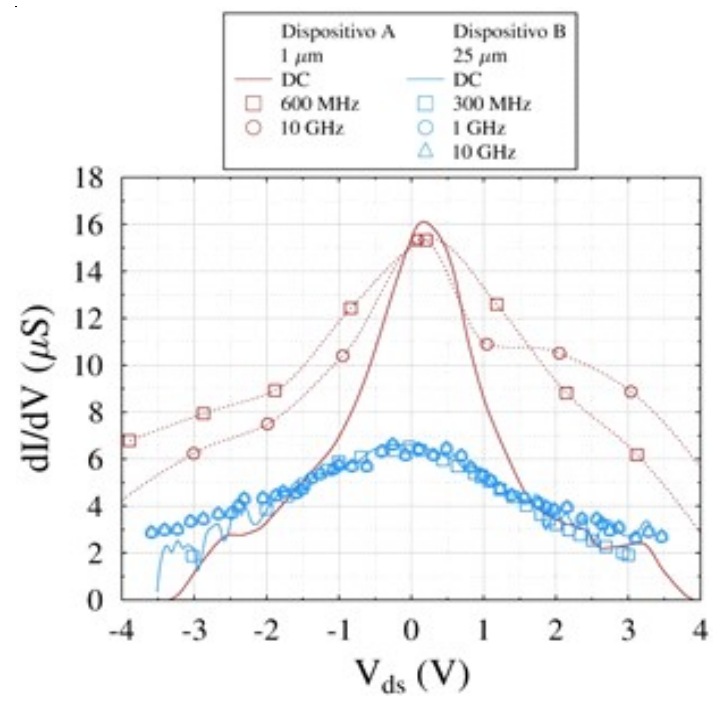

Fig. 2. Conductancia en AC y DC del dispositivo (A) con un SWCNT metálico de longitud $1 \mu \mathrm{m}$ y del dispositivo (B) con un SWCNT metálico de longitud $25 \mu \mathrm{m}$. Datos tomados de [12]. tura basada en 3 contactos los cuales se distribuyen de manera tierra-señal-tierra) con arreglo efectivo de un CNT de longitud $20 \mu \mathrm{m}$ y sin arreglo, en la Fig. 3 se muestra la magnitud de los parámetros $S_{11}$ y $S_{21}$ que se obtuvieron. Los parámetros $S$ son una herramienta necesaria que en los equipos de medición nos indicará el grado de acoplamiento de la señal en los dispositivos, siendo el parámetro $S_{11}$ (coeficiente de reflexión) el que indicará la cantidad de potencia reflejada de la señal que incide en la entrada y siendo el parámetro $S_{21}$ (coeficiente de transmisión) el que denotará la cantidad de potencia que se logra transmitir de la entrada a la salida del dispositivo.

La disminución del efecto pelicular debido a la inductancia cinética se ve reflejado por la mejora de los parámetros $S_{11}$ y $S_{21}$ a medida que la frecuencia es incrementada.

Al final de este artículo se propone que los CNT pueden ser aplicados como interconectores en tecnología de integración a gran escala (VLSI por sus siglas en inglés) o como inductores "on-chip".

El comportamiento de películas de grosores de $25 \mathrm{~nm}$ - $60 \mathrm{~nm}$ de CNT metálicos fue el principal objetivo a estudiar de ma-

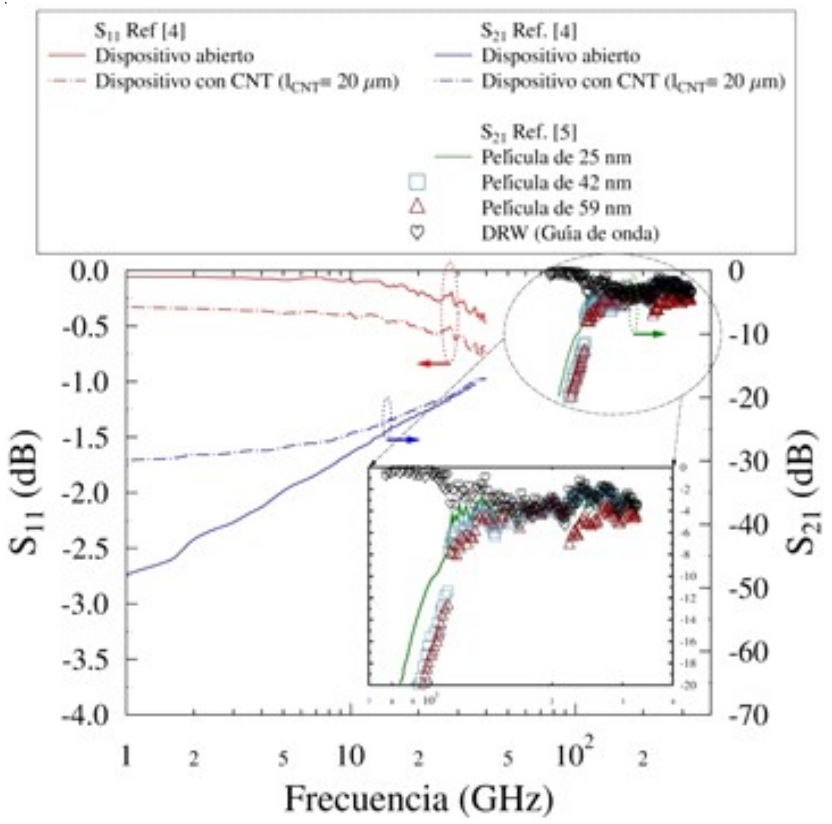

Fig. 3. Magnitudes de los parámetros medidos $S_{11}$ y $S_{21}$ para la guía de onda coplanar propuesta en [13] con y sin CNT. Magnitudes del parámetro medido $S_{21}$ para diferentes grosores de películas de CNT puestas en una guía de onda propuesta en [14]. Datos tomados de [13] y [14] respectivamente. 
nera experimental mediante el uso de una guía de onda dieléctrica en [14], para un rango de frecuencias de estudio de 75 a $320 \mathrm{GHz}$. Aunque ambos tipos de CNT (metálicos y semiconductores) se implementaron en las películas, las contribuciones de los CNT semiconductores fueron despreciadas en los cálculos. En la Fig. 3 se pueden apreciar los datos del parámetro $S_{21}$ obtenidos de las películas delgadas colocadas en la guía de onda coplanar y medidas por un VNA (Analizador de Redes vectorial por sus siglas en inglés). Se observa que debido al cambio de calibración hecho para la configuración de los diferentes accesos de la guía de onda (modelos WR-XX) existe una diferencia abrupta en los valores medidos del parámetro $S_{21}$. Así mismo, se percibió que dichas pérdidas (por transmisión) disminuyen al incrementar la frecuencia. Otra aplicación de estas películas de CNT es como atenuadores para frecuencias menores a $90 \mathrm{GHz}$.

La factibilidad de utilizar arreglos de CNT (modelo CG-200 de southwest technology) como interconectores para circuitos tipo flip-chip se expone en [15], así como su desempeño en comparación con los interconectores con base de oro. En la Fig. 4 [15] se muestran los parámetros $S$ obtenidos en este artículo, se puede distinguir que el coeficiente de transmisión disminuye a mayores frecuencias. Los interconectores a base de CNT entre el dispositivo "Flip-chip" mostraron tener mayores pérdidas por inserción que los interconectores con base de oro debido a la resistencia de los CNT, sin embargo, estas pérdidas por inserción son ligeramente mayores (en $0.3 \mathrm{~dB}$ ) a las del oro, sin mencionar que potencialmente en altas frecuencias dichas pérdidas pueden ser menores debido a la inductancia cinética de los CNT que aminorará el efecto pelicular [15].

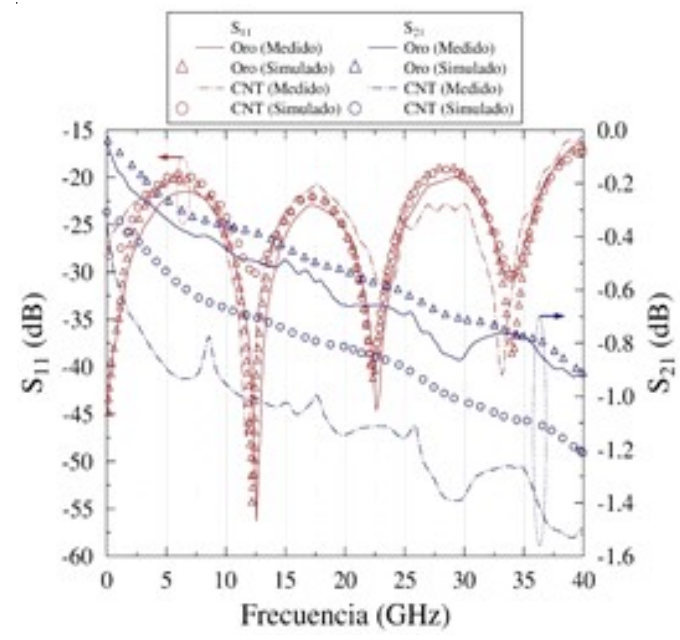

Fig. 4. Parámetros $S$ del circuito flip-chip utilizando como interconectores oro/CNT. Datos tomados de [15].
Los interconectores con base en CNT ofrecieron pérdidas por retorno (coeficiente de reflexión o parámetro $S_{11}$ ) mejores a $15 \mathrm{~dB}$ hasta $40 \mathrm{GHz}$ y pérdidas por inserción (coeficiente de transmisión o parámetro $S_{21}$ ) aproximadamente $0.3 \mathrm{~dB}$ mayores a las del oro convencional, convirtiéndolos en un material con desempeño similar al oro.

Una investigación teórica sobre el impacto de la concentración de CNT metálicos (el estudio abarcó desde una fracción metálica o $F_{m}=1 / 3$ de CNT metálicos del total del arreglo, hasta todo el arreglo) en arreglos implementados en altas frecuencias es presentada en [16]. En la Fig. 5 [16] se muestra la gráfica de la profundidad de penetración del efecto pelicular a diferentes frecuencias utilizando la ecuación de la onda viajera (ecuaciones de Maxwell). Como se observa en la Fig. 5 dicha investigación revela que el efecto pelicular en los arreglos de CNT de multipared, o mejor conocidos por su abreviatura en inglés MWCNT, es significativamente reducido en altas frecuencias (especialmente en frecuencias mayores a $50 \mathrm{GHz}$ ) en comparación con CNT de pared simple (SWCNT metálicos y semiconductores) e inclusive conductores de cobre convencionales, lo que se traduciría en valores de impedancia menores y constantes de un interconector en aplicaciones en altas frecuencias.

El desempeño eléctrico de una vía transversal de silicio (TSV) compuesta por CNT puede verse afectado por la variación de la inductancia cinética en el canal y degradar la conductividad en altas frecuencias, por ello la conductividad eléctrica de una TSV compuesta por CNT puede ser mejorada de dos maneras [17]: al depositar cobre en la misma vía o aumentar la cantidad de CNT (que a su vez aumentará el $F_{C N T}$ o la cantidad

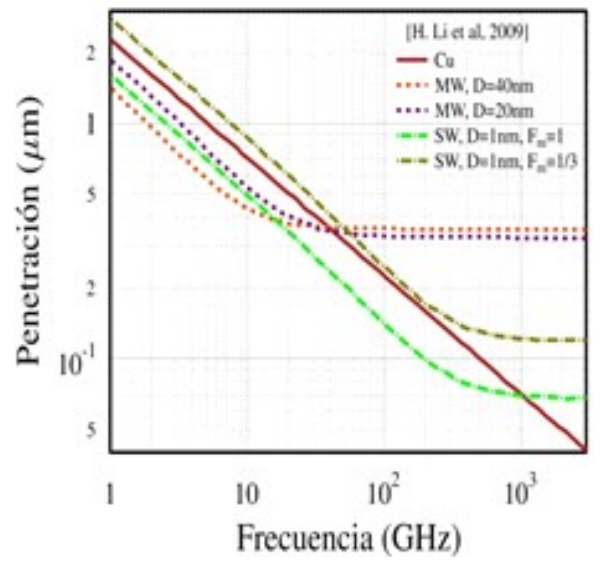

Fig. 5. Profundidad de penetración del efecto pelicular en diferentes tipos de CNT, así como en Cu, en función de la frecuencia. Datos tomados de [16]. 

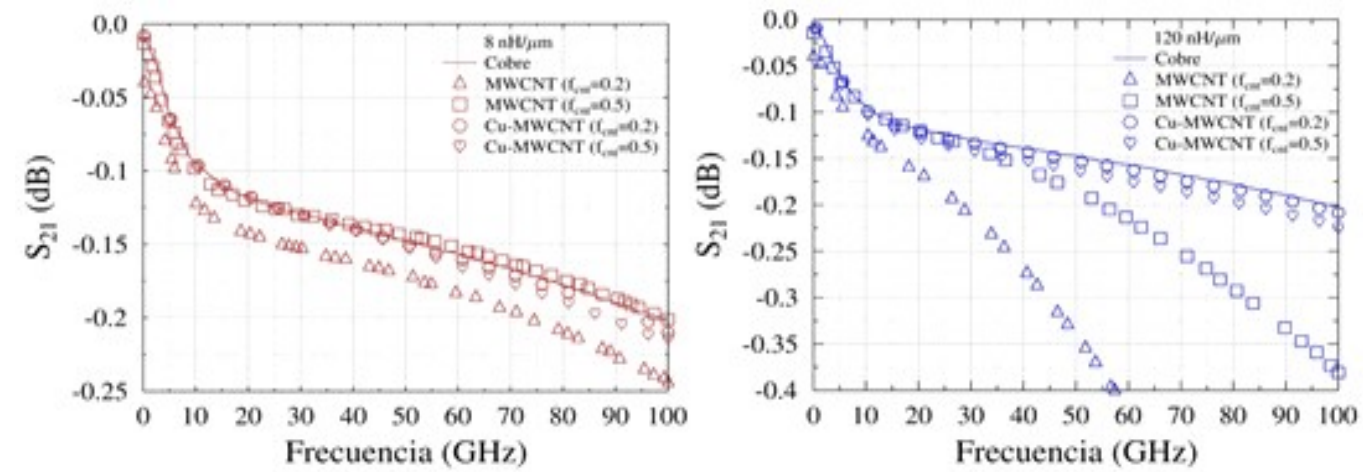

Fig. 6. Coeficiente de transmisión $S_{21}$ para un par de MWCNT y Cu-MWCNT embebidos en vías transversales de silicio con impedancias cinéticas por canal definidas. Datos tomados de [17].

de CNT embebidos). En la Fig. 8 [17] se puede observar como el coeficiente de transmisión $\left(S_{21}\right)$ de un par de MWCNT y Cu-MWCNT embebidos en TSV mejora y se vuelve cercano al comportamiento de una TSV de cobre al incrementar la cantidad de CNT que se embeben en ella o bien al añadir cobre a la TSV junto a los CNT .Los valores de inductancia propuestos fueron retomados de dos reportes (no mencionados aquí) los cuales sugieren como límite inferior la inductancia cinética producida por un solo canal que es aproximada a $8 \mathrm{nH} / \mu \mathrm{m}$ y como límite superior $120 \mathrm{nH} / \mu \mathrm{m}$ que es el punto reportado donde el desempeño de los arreglos de CNT se empieza a volver impredecible.

En conclusión, con todas las propiedades descritas en esta sección, los CNT serán candidatos a ser inteconectores por ofrecer pérdidas por retorno (coeficiente de reflexión o $S_{11}$ ) y pérdidas por inserción $\left(S_{21}\right.$ o coeficiente de transmisión) casi iguales que las del oro convencional. Serán factibles cuando las frecuencias a utilizar sean mayores a $50 \mathrm{GHz}$, pueden de igual forma, usarse como atenuadores a frecuencias menores de $90 \mathrm{GHz}$, tomando en cuenta una pureza del arreglo de los CNT del 100\% (o fracción metálica $F_{m}=1$ ). Sin embargo, mejores resultados de desempeño en altas frecuencias y menor penetración del efecto pelicular pueden ser alcanzados con arreglos de MWCNT y este a su vez usando como interconectores TSV con una cantidad considerable de MWCNT embebidos $\left(F_{C N T}\right)$ o con cobre en la misma vía (Cu-MWCNT). Un estudio detallado de estas estructuras queda fuera del enfoque de este artículo, sin embargo, el lector interesado en ellas puede consultar [22].

\section{Modelo compacto y extracción de valores para cada uno de sus elementos}

A continuación, se describirá la metodología usada en [13] para la obtención de un modelo que describa el comportamiento en altas frecuencias de un interconector con base en
CNT, tomando como premisa la deducción de un circuito eléctrico RLC equivalente.

Tal como se citó en la sección 2, un factor importante a tomar en cuenta para la fabricación o modelado de un interconector con base en CNT es la inductancia cinética. El primer paso realizado en [13] fue calcular teóricamente la inductancia cinética y la contribución de esta a la inductancia total del arreglo. Para esta estimación tomaron en cuenta un arreglo de MWCNT con $N$ elementos, con un diámetro promedio de $7.5 \mathrm{~nm}$ por MWCNT, un largo del arreglo de $10 \mu \mathrm{m}$ y un ancho del arreglo $3.5 \mu \mathrm{m}$.

Para el cálculo de la inductancia cinética del CNT en el arreglo tomaron en cuenta lo siguiente: cuando la longitud del CNT es mayor a la trayectoria libre media (MFP por sus siglas en inglés) del electrón, la inductancia cinética será proporcional a la longitud del CNT e inversamente proporcional al número de CNT en paralelo, así como se muestra en la ecuación (1) [16]:

$$
L_{K_{C N T}}=\frac{L_{K}}{N} \times l
$$

Donde, $L_{K_{C N T}}$ es la inductancia cinética producida por el arreglo de CNT, $l$ es la longitud del arreglo, $N$ es el número de CNT en el arreglo y $L_{K}$ es la inductancia cinética por unidad de longitud, la cual es aproximada a $8 \mathrm{nH} / \mu \mathrm{m}$ [16]. Cabe resaltar que, aunque no hacen mención del valor de $N$, aluden que fue estimado mediante el uso de un modelo preestablecido, el cual no es discutido en el presente artículo.

Por otro lado, para estimar la inductancia total $\left(L_{T o T}\right)$ del arreglo utilizaron un circuito equivalente de elementos parciales (tampoco estudiado en el presente artículo), descubriendo que el $98.3 \%$ de la inductancia total del arreglo es generada de la inductancia cinética del arreglo de CNT $\left(L_{K_{C N T}}\right)$. Conclu- 
yeron que la inductancia total del dispositivo por fabricar será en gran parte a la contribución hecha por la inductancia cinética.

El siguiente paso que realizaron fue fabricar diferentes dispositivos (guías de onda coplanares) con CNT de longitudes efectivas (o longitudes teóricas dado que en la implementación no se tiene un control sobre el tamaño exacto de los CNT) diferentes de 10, 20 y $30 \mu \mathrm{m}$, sin mencionar los dispositivos abiertos ( $\sin \mathrm{CNT}$ ) fabricados que utilizaron para desembeber parámetros parásitos. Los dispositivos que elaboraron fueron caracterizados en corriente directa (DC) y en frecuencia (RF).

Para lograr caracterizar dichos dispositivos en corriente directa (DC) aplicaron un voltaje de -1 a $1 \mathrm{~V}$ entre las terminales de 3 de los diferentes dispositivos fabricados (guías de onda coplanares) con CNT de longitudes efectivas diferentes (10, 20 y $30 \mu \mathrm{m}$ ) para medir las resistencias diferenciales totales $\left(R_{T o T}=R_{C}+R_{C N T} \sim R_{D C}\right)$ de cada uno. Midieron de 3 a 10 muestras de resistencia para cada dispositivo y con los valores promedio para cada arreglo bosquejaron la ecuación (2) [13] la cual describe los valores posibles que pudiera tomar $R_{\text {TоT }}$ para diferentes longitudes efectivas en el arreglo de CNT,

$$
R_{D C}=(1267.9 \pm 1.482) \Omega+(90 \pm 8.4) \Omega / \mu \mathrm{m} \cdot l_{C N T}
$$

Gracias a la ecuación (2) se pueden distinguir 2 diferentes componentes en resistencia: uno que se mantiene constante y otro que variará por la longitud de los CNT, en otras palabras, se podrá decir que la primera parte de la ecuación (2) $(1267.9 \pm 148.2) \Omega$ es producida por el contacto entre los CNT y el contacto del dispositivo $\left(R_{C}\right)$, mientras que la segunda parte de la ecuación (2) $(90 \pm 8.4) \Omega / \mu \mathrm{m} \cdot l_{C N T}$ es producida meramente por la longitud de los $\mathrm{CNT}\left(R_{C N T}\right)$. La fluctuación de valores posibles en $R_{D C}$ es debido a que ésta varía en voltajes de polarización $V>0.4 \mathrm{~V}$, estimando de esta manera un valor máximo de voltaje el cual ayudo para la caracterización en frecuencia.

Para la caracterización en frecuencia (RF) midieron la magnitud de la impedancia total del dispositivo con CNT de longitud efectiva de $20 \mu \mathrm{m}$, luego mediante métodos de desembebido (no mencionados en este artículo, aunque se intuye que fue mediante la medición del dispositivo abierto con longitud de $20 \mu \mathrm{m}$ ) eliminaron los componentes parásitos (provenientes del pad) de la impedancia. Con la impedancia obtenida propusieron un circuito RLC (véase Fig. 7) y valores de cada uno de sus componentes, para simular el circuito por medio del software de diseño electrónico Advance Design System (ADS) y obtener una impedancia, la cual se ajustará a la impedancia medida. Los valores que consideraron para la

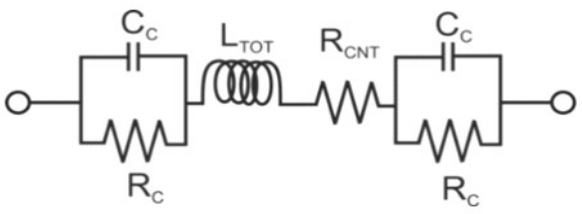

Fig. 7. Circuito eléctrico equivalente RLC propuesto para un arreglo de CNT. Circuito tomado de [13].

implementación en ADS para una guía de onda coplanar de $20 \mu \mathrm{m}$ de largo, se muestran en la Tabla 1 [13].

Retomando los componentes del circuito RLC de la Fig. 2, $R_{C}$ y $C_{C}$ son las resistencias y capacitancias producidas por la $R_{C N T}$ es la resistencia producida por el CNT y $L_{T o T}$ es la inductancia total del dispositivo.

Para la obtención de $L_{T o T}$, la cual como demostraron es mayoritariamente la inductancia cinética del arreglo de CNT, hicieron un procedimiento análogo al del cálculo de la resistencia (utilizando las guías de onda coplanares con diferentes longitudes de CNT se estimaron los valores, se tomaron muestras y se calcularon los valores de inductancias) y se obtuvo la ecuación (3) [13]:

$$
L_{T o T}=(-0.05 \pm 0.12) \mathrm{nH}+(0.105 \pm 0.005) \mathrm{nH} / \mu \mathrm{m} \cdot l_{C N T}
$$

Para finalizar, cabe señalar que el valor de la capacitancia $C_{C}$ fue estimado puramente por medio de los valores medidos de impedancia y sus métodos de desembebido.

\section{Estudio y simulación en altas frecuencias del modelo compacto}

Haciendo uso de los datos proporcionados de la Tabla 1 y con el circuito eléctrico equivalente (véase Fig. 7), se obtuvo la ecuación general de impedancia (no proporcionada en [13]) para el modelo propuesto en [13]. El resultado de haber aplicado las leyes de Kirchhoff al circuito RLC es la ecuación propuesta (4):

$$
z_{t o t}=R_{C N T}+\frac{2 R_{c}}{1+R_{C}^{2} \omega^{2} C_{C}^{2}}+j \omega\left[L_{t o t}-\frac{2 R_{C}^{2} C_{C}}{1+R_{C}^{2} \omega^{2} C_{C}^{2}}\right]
$$

Tabla 1. Valores de los parámetros del modelo RLC para un arreglo de MWCNT de $20 \mu \mathrm{m}$ de largo (datos tomados de [13]).

\begin{tabular}{lcccc}
\hline Parámetros & $\mathbf{R c}(\mathbf{\Omega})$ & $\mathbf{C c}(\mathbf{f F})$ & $R_{C N T}(\mathbf{\Omega})$ & $L_{\text {ToT }}(\mathbf{n H})$ \\
Valores & 375 & 228 & 1770 & 2.2 \\
\hline
\end{tabular}


La ecuación (4) brinda una descripción del comportamiento en frecuencia de la impedancia total del interconector con base en CNT, la cual utiliza como parámetros valores que pueden ser conocidos por medio de las ecuaciones (1), (2) y (3), sencillamente sabiendo la longitud del arreglo de CNT que fungirán como interconector.

Uno de los motivos para el cálculo de una función de impedancia descriptiva del dispositivo en función de la frecuencia. es analizar a detalle el circuito presentado en la Fig. 7 y observar conductas que con la sola implementación en un software de modelado y simulación (como lo es ADS) no se podrían percibir en primera instancia, por ejemplo, observamos en la ecuación (4) que en altas frecuencias la impedancia que predominará en el dispositivo será la producida por los CNT. Para validar la veracidad de la ecuación (4) que se propone en el presente artículo se realizó una comparativa con los valores de impedancia reportados en [13] y con el software de diseño electrónico (ADS), graficando las impedancias en un solo plano y utilizando como referencia los valores de cada componente dados en la Tabla 1 , tal como se muestra en la Fig. 8.

Gracias a la Fig. 8 se corroboró que la ecuación (4) puede describir de manera precisa el comportamiento de impedancia del dispositivo en altas frecuencias, sin embargo, indagaremos más sobre las características de dicha impedancia para lograr entender la fenomenología detrás de este dispositivo. La parte real e imaginaria de la impedancia del dispositivo fueron separadas y graficadas tal como se muestra en la Fig. 9.

Mediante la Fig. 9 se pueden observar los 2 comportamientos distintos que rigen la impedancia total: del lado de la parte

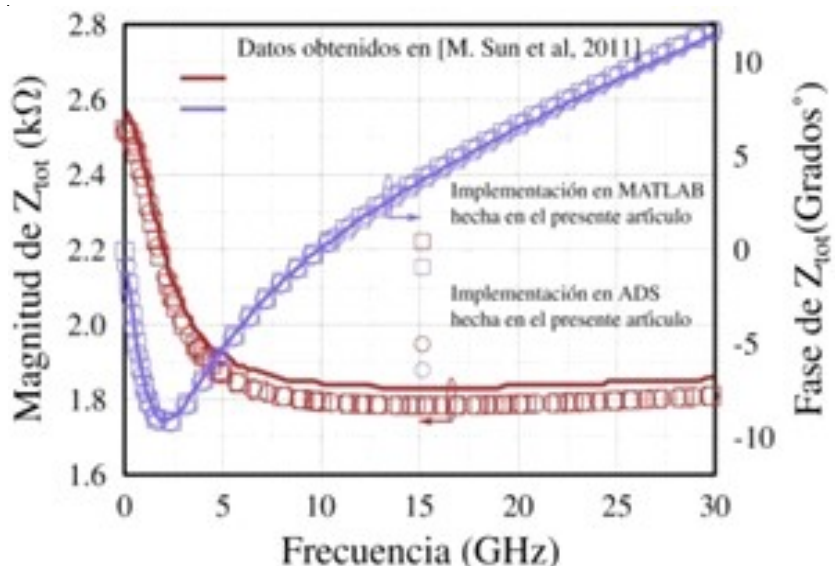

Fig. 8. Comparación entre valores de impedancia obtenidos con la ecuación propuesta (4), implementado ADS y MATLAB contra los $v$ alores reportados en [13].

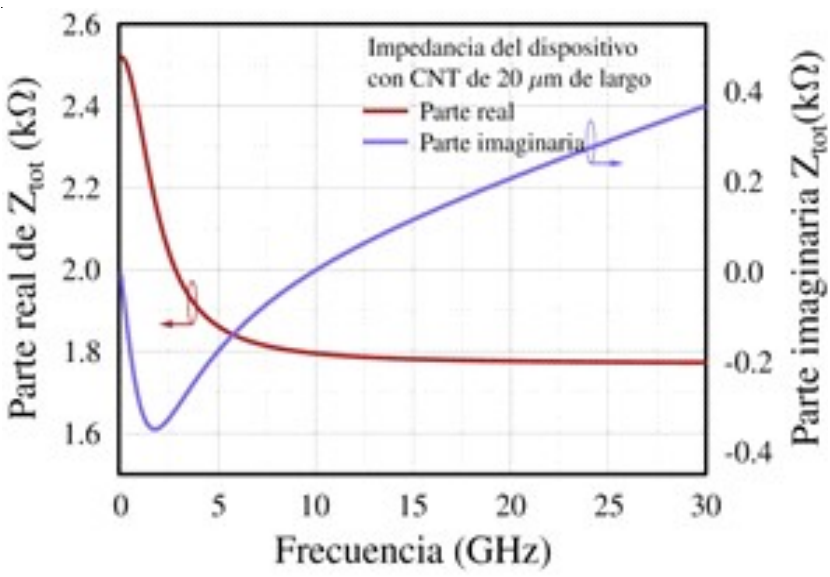

Fig. 9. Comportamiento de la parte real e imaginaria de la impedancia del dispositivo.

real y de la parte imaginaria del dispositivo. Para el caso de la parte real, se observa que el dispositivo al inicio (frecuencias $<10 \mathrm{GHz}$ ) presentará una resistencia de mayor magnitud, producida por el proceso de acoplamiento resistivo entre los contactos del dispositivo y el CNT, que su resistencia final (después del acoplamiento o a frecuencias $>10 \mathrm{GHz}$ ), además dicha resistencia final se mantendrá constate y estará definida en su mayoría por el CNT y su longitud. Para el caso de la parte imaginaria de la impedancia (reactancia) se observa que adquiere valores negativos a frecuencias menores a $10 \mathrm{GHz}$ (al igual que su contraparte real) lo que indica un proceso de acoplamiento capacitivo entre los contactos y el CNT, para después incrementar debido a la inductancia cinética producida por el CNT.

Para seguir complementando el estudio realizado en [13], se propusieron valores de longitud de arreglos de CNT con largos de 10, 20, 30 y $40 \mu \mathrm{m}$ hasta frecuencias aproximadas a los $500 \mathrm{GHz}$ y utilizando las ecuaciones (2), (3) y (4) se estimó el valor de las impedancias características para cada interconector. Cabe recalcar que en [13] solamente se trabajó en la longitud efectiva del arreglo de $20 \mu \mathrm{m}$ para el cálculo de la impedancia total, sin mencionar que el estudio en frecuencias que se hace solo abarcó hasta los $30 \mathrm{GHz}$ como ya se había observado anteriormente en la Fig. 8.

En la Fig. 10 se hizo una comparativa del comportamiento en frecuencia de los valores de impedancia para diferentes largos de CNT, anexando además el de un interconector a base de cobre [16] de $500 \mu \mathrm{m}$ de largo con dimensiones $2 \times 1 \mu \mathrm{m}$.

Se observa en la Fig. 10 que el interconector con un largo de $10 \mu \mathrm{m}$ es el que menor impedancia generará y no solo eso, es 


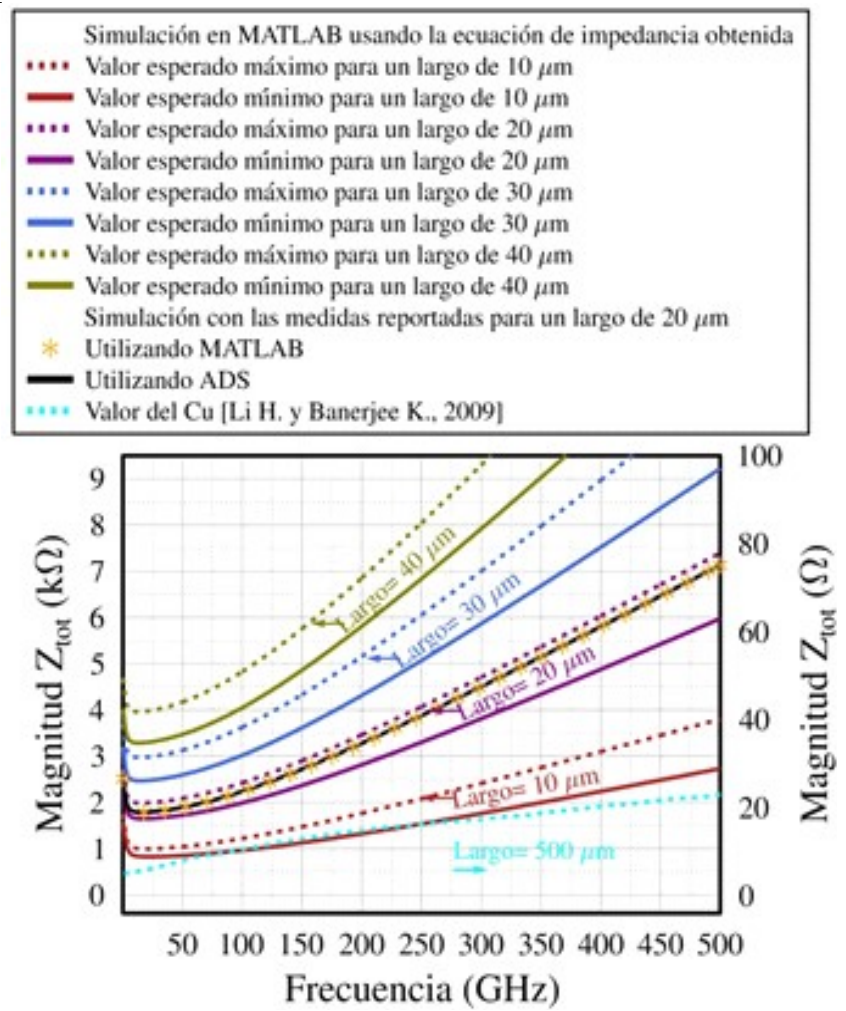

Fig. 10. Comportamiento en frecuencia de la impedancia producida por diferentes longitudes de CNT en un dispositivo contra la impedancia producida por el cobre [16] con medidas $2 \times 1 \mu \mathrm{m}$.

el que menos incrementará su magnitud de impedancia en altas frecuencias, por tanto, y acorde al modelo propuesto en [13], si se desea implementar un interconector con base en CNT deberá ser a longitudes $<<10 \mu \mathrm{m}$. Por otro lado, el comportamiento de la impedancia total de un interconector de cobre tenderá a subir conforme aumente la frecuencia, mientras que el del CNT primero tenderá a bajar (mientras se acopla los contactos del dispositivo con los CNT), mantenerse constante (después de haber alcanzado el acoplamiento) y por último volver a subir (debido al ligero pero constante incremento producido por la inductancia), inclusive rebasando sus valores de impedancia inicial.

Para definir el comportamiento de las impedancias obtenidas se separaron (como se hizo en el análisis de la Fig. 9) en sus respectivas partes reales e imaginarias, se graficaron y se interpretaron los valores obtenidos.

Como se puede apreciar en la Fig. 11 el comportamiento en altas frecuencias de la parte real de la impedancia del dispositivo indica la ya antes mencionada resistencia de acoplamien-

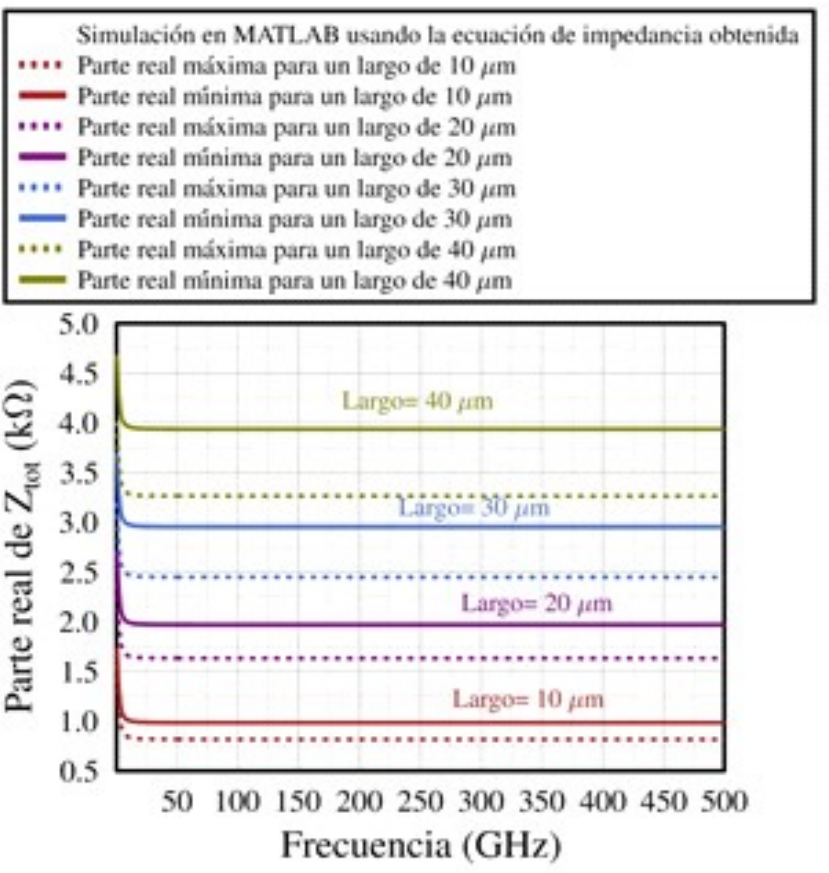

Fig. 11. Comportamiento en frecuencia de la parte real de la impedancia producida por diferentes longitudes de CNT.

to en bajas frecuencias $(<10 \mathrm{GHz})$ y después de alcanzada su frecuencia de acoplamiento se percibe que el valor se mantiene constante y no solo eso, también dicho valor final constante alcanzado está definido por el largo del CNT, es decir, se corrobora que a mayor longitud del CNT la impedancia del dispositivo será mayor.

Por otro lado, como se distingue en el comportamiento en altas frecuencias en la Fig. 12 de la parte imaginaria de la impedancia del dispositivo, aparece una frecuencia de acoplamiento la cual al ser alcanzada produce un aumento gradual en la impedancia (por la inductancia cinética), además dicho incremento logra impedancias mucho mayores que las de la parte real. También se concluye que la parte imaginaria contribuye en gran medida a la impedancia total del dispositivo.

Como ejercicio y poder bosquejar de manera sencilla el impacto de la correcta implementación de los CNT se grafica en la Fig. 13 la impedancia del dispositivo sin tomar en cuenta las contribuciones por el acoplamiento entre los contactos (resistencia y acoplamiento) y CNT, es decir, se encuentra acoplado $\left(R_{C}=50 \Omega\right.$ y $\left.C_{C}=0\right)$ el dispositivo y el CNT.

Se descubre que al acoplar los contactos del dispositivo con el CNT se obtiene una conducta muy parecida a la del interconector de cobre convencional. 


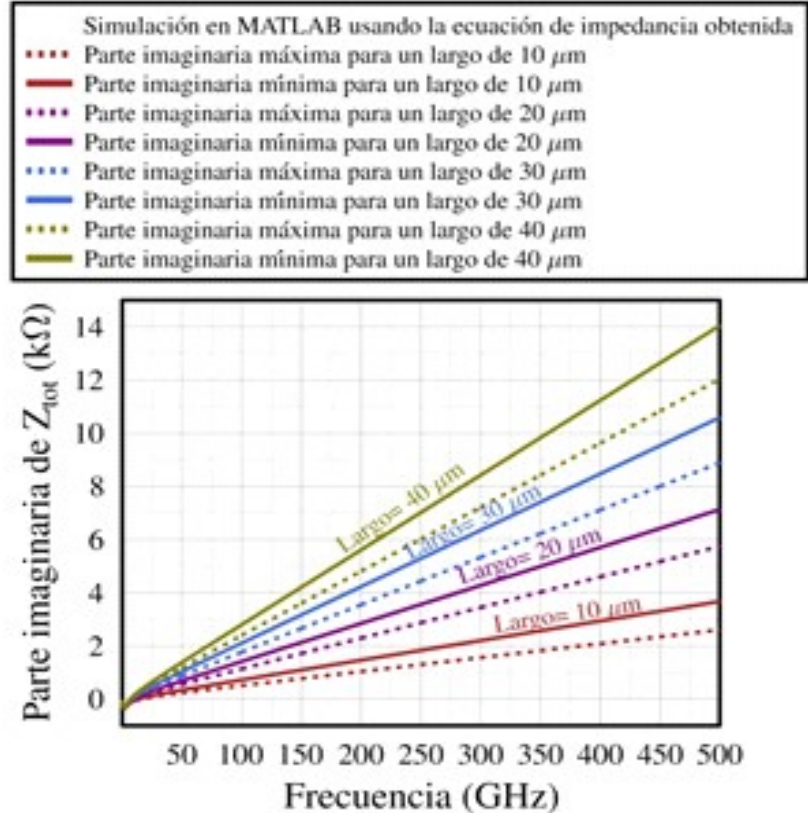

Fig. 12. Comportamiento en frecuencia de la parte real de la impedancia producida por diferentes longitudes de CNT.

Después, mediante ADS y utilizando el circuito de la Fig. 7 se simularon los parámetros $\mathrm{S}$ de un interconector de CNT con longitud $20 \mu \mathrm{m}$ y se compararon con los parámetros $S$ reportados en [13] tal como se muestra en la Fig. 14.

Podemos advertir en la Fig. 14 que los valores reportados en [13] de los parámetros $S$ con los simulados en ADS son pare-

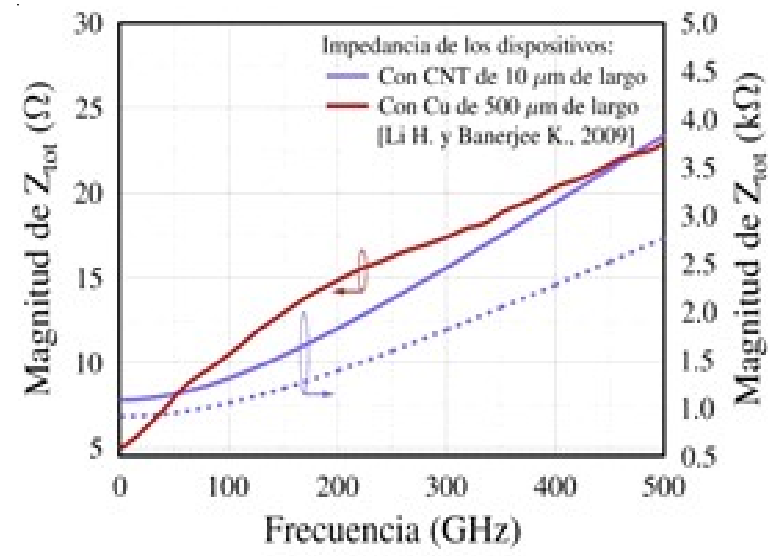

Fig. 13. Comportamiento en frecuencia de la impedancia producida por un CNT acoplado con el dispositivo contra la impedancia producida por el cobre [16] con medidas $2 \times 1 \mu \mathrm{m}$.

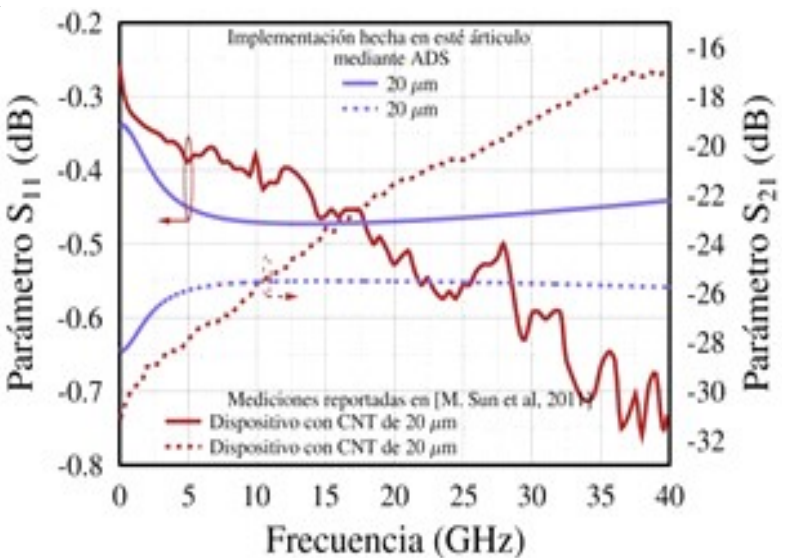

Fig. 14. Comparación del comportamiento en frecuencia de los parámetros S simulados en ADS y medidos en el dispositivo [13].

cidos, sin embargo, dicha discrepancia de valores pudiera ser producida por defectos del dispositivo, por ejemplo y como se menciona en [13], solo unos cuantos CNT hacen contacto con el pad. Otro efecto mencionado que probablemente afectó las mediciones de los parámetros $S$, también mencionado en [13], sea la dominancia de la capacitancia de acoplamiento del arreglo en altas frecuencias.

Debido a la discrepancia de los valores de los parámetros $S$ obtenidos mediante el software de diseño electrónico (ADS) y los mostrados en [13], será necesario deducir las ecuaciones que rigen los comportamientos de los parámetros $S$ en altas frecuencias y determinar el valor real de los componentes del circuito RLC.

Primero, utilizando el circuito de la Fig. 7 se agrupan los componentes en 3 bloques generales obteniendo impedancias equivalentes. El primer bloque de impedancia equivalente es producido por el acoplamiento entre el CNT y los contactos del dispositivo y está dada por los valores de $R_{C}$ y $C_{C}$ tal como se muestra en la ecuación (5):

$$
Z_{C}=\frac{R_{C}-R_{C}^{2} j \omega C_{C}}{1+R_{C}^{2} \omega^{2} C_{C}^{2}}
$$

El segundo bloque se compone de la contribución de impedancia producida meramente por el CNT, tal como se muestra en (6):

$$
Z_{C N T}=R_{C N T}+j \omega L_{T o T}
$$


El tercer y último bloque de impedancia del circuito es de nuevo producido por el acoplamiento entre el CNT y los contactos del dispositivo, por tanto, está dado por la ecuación (5). Cabe destacar que la suma de los valores de $2 Z_{C}$ y $Z_{C N T}$ da como resultado el valor de $Z_{T o T}$ (ecuación 4).

Con el uso de los bloques de impedancias y sus respectivas ecuaciones (5) y (6) se simplificará el análisis del circuito. Al tener un circuito compuesto únicamente por 3 impedancias en serie se hará uso de los parámetros $A B C D$ (de transmisión) dadas las siguientes 3 circunstancias: al ser un circuito pasivo se considerará como recíproco, por ello la relación $A D-B C=1[18]$ de los parámetros se cumplirá. No tendrá ganancia de entrada-salida o salida-entrada (será simétrico) dado que los elementos son pasivos, sin mencionar que además la posición de los componentes y sus valores de circuito son los mismos en ambos puertos, por tanto, existe una relación $A=D[18]$ de los parámetros de transmisión. Es sencillo el análisis usando los parámetros $A B C D$ por la topología del circuito.

A continuación, la ecuación (7) describe el comportamiento del parámetro $A$ :

$$
[A]=\left[Z_{C N T}+2 Z_{C}\right]
$$

el comportamiento del parámetro $B$ estará definido por la ecuación (8):

$$
[B]=\left[Z_{C N T}+2 Z_{C}\right]^{2}-1
$$

el parámetro $D$ se calculará de la misma forma del parámetro $A$ (dado a su simetría mencionada) tal como se ve en la ecuación (9):

$$
[D]=\left[Z_{C N T}+2 Z_{C}\right]
$$

y, por último, el parámetro $C$ el cual estará dado por la ecuación (10):

$$
[C]=[1]
$$

Con los valores de cada uno de los elementos de la matriz $A B C D$, se normalizan los valores de $A B C D$ tomando en cuenta una impedancia de carga y una impedancia de la fuente de $50 \Omega$ (la fuente y la carga están acopladas al dispositivo) y utilizando las ecuaciones de equivalencia a parámetros $S$ [18] (no discutidas en esté artículo) se calculan los parámetros $S$. Para el parámetro $S_{11}$ la ecuación (11) define su comportamiento en altas frecuencias y está dada por:

$$
S_{11}=\frac{\left[Z_{C N T}+2 Z_{C}\right]^{2}-2501}{\left[Z_{C N T}+2 Z_{C}\right]^{2}+100\left[Z_{C N T}+2 Z_{C}\right]+2499}
$$

Tabla 2. Valores de los parámetros del modelo RLC propuestos para la optimización de los parámetros $S$.

\begin{tabular}{ccccc}
\hline Parámetros & $\mathbf{R c}(\boldsymbol{\Omega})$ & $\mathbf{C c}(\mathbf{f F})$ & $R_{C N T}(\boldsymbol{\Omega})$ & $L_{T o T}(\mathbf{n H})$ \\
$\mathrm{S}_{11}=\mathrm{S}_{22}$ & 770 & 100 & 900 & 2.2 \\
$\mathrm{~S}_{21}=\mathrm{S}_{12}$ & 1050 & 90 & 1770 & 1.9 \\
\hline
\end{tabular}

mientras que para el parámetro $S_{21}$ la ecuación(12) definirá su comportamiento en altas frecuencias.

$$
S_{21}=\frac{100}{\left[Z_{C N T}+2 Z_{C}\right]^{2}+100\left[Z_{C N T}+2 Z_{C}\right]+2499}
$$

Con los parámetros $S$ medidos del dispositivo presentados en [13] y manipulando mediante MATLAB los parámetros de las ecuaciones (11) y (12) en valores cercanos a los reportados en la Tabla 1, se logra optimizar el dispositivo, encontrando valores que se proponen en la Tabla 2, los cuales hacen que los parámetros $S$ teóricos se asemejen al comportamiento práctico del dispositivo.

Utilizando los valores propuestos en la Tabla 2 y las ecuaciones (11) y (12) se comparan nuevamente los valores de los parámetros $S$ con los reportados en [13], tal como se muestra en la Fig. 15.

Se observa en la Fig. 15 que se logra obtener un comportamiento más parecido al reportado prácticamente, también gracias a la Tabla 2 se confirma que los parámetros que más cam-

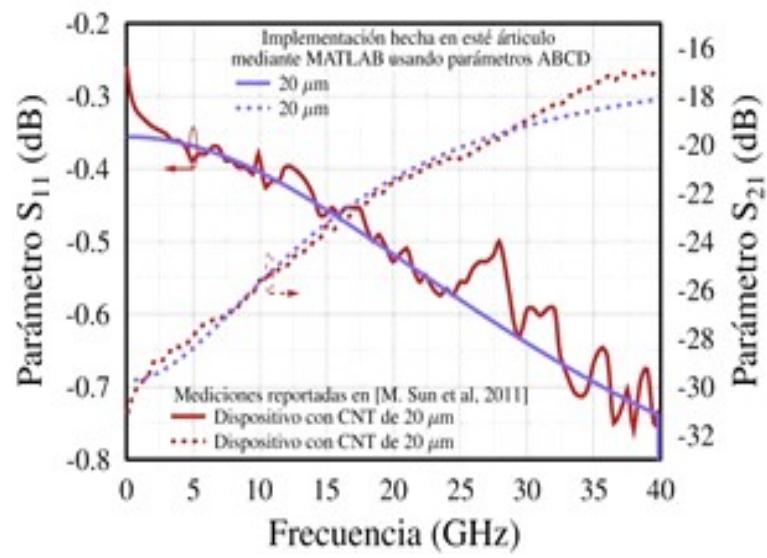

Fig. 15. Comportamiento en frecuencia de los parámetros $\mathrm{S}$ medidos en [13] y de los parámetros $S$ calculados con las ecuaciones (11) y (12) después de proponer los valores de la Tabla 2 para cada componente del modelo. 
biaron en el circuito RLC, tomando como base la Tabla 1, fueron la capacitancia y resistencia de acoplamiento en comparación con la resistencia e inductancia producida por los CNT, confirmando que los defectos de fabricación estarán en los contactos del dispositivo y que todas las ecuaciones calculadas en esté artículo podrán bosquejar el comportamiento de los CNT para este modelo propuesto.

Como conclusión de la sección, se obtuvieron ecuaciones que describen el comportamiento en frecuencia de la impedancia y de los parámetros $S$ del modelo propuesto en [13], extendiendo el mismo a frecuencias superiores a $30 \mathrm{GHz}$. Se analizó la impedancia característica del dispositivo y se concluyó que la parte real (correspondiente a la resistividad) estará dada, en frecuencias menores a $10 \mathrm{GHz}$, por la resistencia de acoplamiento para pasada esta frecuencia comenzar a disminuir y ser meramente la resistencia del CNT, la cual se mantuvo constante en todo el rango estudiado $(1 \mathrm{GHz}-500 \mathrm{GHz})$ y depende de la longitud del CNT. Por otro lado, en la parte imaginaria de la impedancia se descubrió que en frecuencias menores a $10 \mathrm{GHz}$, la capacitancia producida por el acoplamiento es dominante. A frecuencias mayores a $10 \mathrm{GHz}$ sin embargo, la inductancia producida por los CNT es más significativa. Este fenómeno está ligado directamente a la longitud de los CNT, i. e., la parte imaginaria de la impedancia está dominada por reactancias capacitivas o inductivas dependiendo de la frecuencia de trabajo.

Se percibe que al acoplar de manera correcta los contactos del dispositivo con el CNT, se obtendrá un comportamiento cuasi-similar al del cobre.

Se descubrió que el comportamiento de este modelo a frecuencias $>10 \mathrm{GHz}$ tiende a un aumento drástico en la impedancia sobre todo en interconectores $>10 \mu \mathrm{m}$, por efectos de la parte imaginaria (inductancia de los CNT) tal como se mencionó previamente.

Por último, se optimizó el modelo proponiendo valores para los elementos del circuito RLC los cuales amoldarán los resultados de la ecuación de los parámetros $S$ (aquí propuesta) con los valores medidos del dispositivo elaborado en [13]. Se descubrió que los datos para los elementos de acoplamiento eran erróneos y se propusieron nuevos, los cuales sugieren defectos en el acoplamiento entre los contactos y el CNT muy probablemente producidos por procesos de fabricación del dispositivo.

\section{Retos tecnológicos}

Como se observó en la sección anterior, tener ecuaciones las cuales nos describan el comportamiento en frecuencias del dispositivo, ya sea en impedancia o en parámetros $S$, no nos asegura poder calcular con precisión el valor real y esto no será debido a la veracidad de las ecuaciones (las cuales se pudieron corroborar), sino por una mala estimación de los elementos del modelo RLC debido a fallas en la fabricación del dispositivo que modificaron los valores originales previstos. En el caso de la fabricación de los contactos se ha tratado de reducir dicha resistencia (de acoplamiento) empleado diferentes materiales [19], no obstante, dicha resistencia es un impedimento para lograr mejores desempeños debido a los fenómenos de dispersión que lo envuelven, que a su vez y como su nombre lo indica, afectan a los parámetros $S$.

Por otro lado, la deposición de CNT en un dispositivo también puede llegar a ser una tarea complicada debido a que no se tiene un control de la cantidad de CNT depositados, sin mencionar los problemas que conlleva orientarlos en la dirección deseada. Aunque hay técnicas, como la dielectroforesis [20], que controla la cantidad de CNT depositados (disminuir o aumentar la concentración de CNT en una solución) y orientación (mediante campo eléctrico), dichas técnicas no son factibles si se desea fabricar dispositivos en masa debido al tiempo que se requiere para realizarlas [20], [21].

\section{Conclusiones}

Se observó en la sección 2 que los CNT se perfilan como candidatos a ser inteconectores por sus propiedades eléctricas (inductancia, impedancia y parámetros de dispersión $S$ ) en altas frecuencias, además de lograr una correcta implementación (parámetros $S$ parecidos a los del oro) como interconectores para circuitos flip-chip. Se descubrió también que el desempeño (conductividad eléctrica) de un interconector de CNT puede ser mejorado añadiendo arreglos de MWCNT o utilizando una TSV con CNT embebidos.

Se retomaron los valores para el modelo RLC propuestos en [13] así como las ecuaciones que se obtienen para describir el comportamiento de cada elemento. Con los datos recopilados del modelo, se encontraron las funciones de impedancia característica y parámetros $S$, logrando con la primera reproducir los valores medidos de impedancia y la cual fue parteaguas para extender el análisis en altas frecuencias.

Se comprendió la fenomenología detrás de la componente de la parte real e imaginaria de la función de impedancia. De lado de la parte real se tienen el comportamiento de la resistencia de acoplamiento y de la resistencia del CNT las cuales, en conjunto, muestran un comportamiento constante al aumentar la frecuencia de la señal, mientras que la parte imaginaria corresponde a la capacitancia de acoplamiento y a la inductancia de los CNT. 
Con la función característica de impedancia del interconector se establecieron diferentes casos de estudio para diferentes largos $(10,20,30$ y $40 \mu \mathrm{m})$ a frecuencias mayores a las reportadas (hasta $500 \mathrm{GHz}$ ), descubriendo que la magnitud de la impedancia característica del interconector tiende a aumentar en frecuencias cercanas a los $10 \mathrm{GHz}$, efecto no reportado antes en el modelo propuesto en [13] y atribuido al acoplamiento entre los contactos del dispositivo y los CNT. Se concluye también, en base en el estudio realizado con el modelo empírico propuesto con anterioridad [13] que la longitud de los CNT en los interconectores es uno de los parámetros que jugará un roll importante, ya que está íntimamente ligado con la impedancia, la cual es una de las características físicas que definen el comportamiento del interconector en altas frecuencia.

Una estimación de los parámetros $S$ pudo ser bosquejada por medio de la simulación en ADS, sin embargo, para obtener valores teóricos más fieles a los prácticos fue necesaria la estimación de las ecuaciones características de los parámetros $S$ (por medio de parámetros $A B C D$ ), localizar valores de los parámetros y proponer valores para los elementos del circuito RLC los cuales optimizaron el modelo.

Valores relativamente bajos de impedancia $(<1 \mathrm{k} \Omega)$ en altas frecuencias podrán ser alcanzados utilizando CNT de largos pequeños $(<10 \mu \mathrm{m})$, en otras palabras, como interconectores locales $(<100 \mu \mathrm{m})$.

Por último, como trabajo a futuro se propone mejorar el modelo RLC añadiendo nuevos elementos del dispositivo que no se toman en cuenta (capacitancias cuánticas, por ejemplo) y compararlo con otros modelos (que consideren arreglos de CNT o TSV con CNT incrustados) para obtener una visión más amplia de su comportamiento en altas frecuencias.

\section{Agradecimientos}

Los autores agradecen al Instituto Politécnico Nacional, a la Secretaria de Investigación y Posgrado mediante el proyecto SIP-20196137, a la Sección de Estudios de Posgrado e Investigación de la Escuela Superior de Ingeniería Mecánica y Eléctrica Unidad Zacatenco, y al Consejo Nacional de Ciencia y Tecnología por el apoyo brindado, así como a la Universitat Autonoma de Barcelona por hacer posible esta colaboración.

\section{Referencias}

[1] A. Todri-Sanial, A. Maffucci, Carbon Nanotube for Interconnects. Process, Design and Applications, Suiza: Springer, 2017, Disponible en: https:/www.springer.com/ gp/book/9783319297446. Consultado: 24 agosto, 2018.
[2] S. Im, N. Srivastava, K. Banerjee, K. E. Goodson, "Scaling Analysis of Multilevel Interconnect Temperatures for High-Performance ICs," IEEE Transactions on Electron Devices, vol. 52, núm. 12, pp. 2710-2719, 2005.

[3] B. Li, T. D. Sullivan, T. C. Lee, D. Badami, "Reliability challenges for copper interconnects," Microelectronics Reliability, vol. 44, núm. 3, pp. 365-380, 2004.

[4] H. Macedo-Zamudio, A. Pacheco-Sanchéz, L. M. Rodríguez-Méndez, E. Ramírez-García, D. Valdéz-Pérez, "Estudio en régimen DC y AC de diodos de nanotubos de carbono para aplicaciones de alta frecuencia," Científica, vol. 23, núm. 2, pp. 91 - 98, julio-diciembre, 2019.

[5] K. Koo, H. Cho, P. Kapur, K. C. Saraswat, "Performance Comparisons Between Carbon Nanotubes, Optical, and $\mathrm{Cu}$ for Future High-Performance On-Chip Interconnect Applications," IEEE Transactions on Electron Devices, vol. 54, no. 12, pp. 3206 - 3215, November, 2007. [en línea]. Disponible en: https://ieeexplore.ieee.org/ document/4383033. Consultado: 29 Agosto, 2018

[6] G. F. Close, S. Yasuda, B. Paul, S. Fujita, H. S. P. Wong, "A $1 \mathrm{GHz}$ Integrated Circuit with Carbon Nanotube Interconnects and Silicon Transistors," Nano Letters, vol 8, núm. 2,pp. 706-709, 2008.

[7] X. Chen, D. Akinwande, K-J. Lee, G. F. Close, S. Yasuda, B. C. Paul, S. Fujita, J. Kong, H. S. P. Wong, "Fully integrated graphene and carbon nanotube interconnects for gigahertz high-speed CMOS electronics," IEEE Transactions on Electron Devices, vol. 57, núm. 11, pp. 3137-3143, 2010.

[8] A. Todri-Sanial, R. Ramos, H. Okuno, J. Dijon, A. Dhavamani, M. Wislicenus, K. Lilienthal, B. Uhlig, T. Sadi, V. P. Georigev, A. Asenov, S. M. Amoroso, A. R. Brown, A. Pender, C. Millar, F. Motzfeld, B. Gotsmann, J. Liang, G. Gonçalves, N. Rupesinghe y K. Teo, "A Survey of Carbon Nanotube Interconnects for Energy Efficient Integrated Circuits," IEEE Circuits and Systems Magazine, pp. 47-62, 2017.

[9] V. Kravchenko, R. Resendíz-Vázquez, "Estimación del ancho de banda para comunicaciones satelitales tomando en cuenta la dispersión en la ionosfera," Científica, vol. 13, núm. 1, pp. 9 - 16, enero-marzo, 2009.

[10] J. J. Plombon, K. P. O'Brien, F. Gstrein, V. M. Dubin, "Highfrequency electrical properties of individual and bundled carbon nanotubes," Applied Physics Letters, vol. 90, pp. 063-106, 2007.

[11] A. Raychowdhury, K. Roy, "Modeling of Metallic Carbon-Nanotube Interconnects for Circuit Simulations and a Comparison with $\mathrm{Cu}$ Interconnects for Scaled Technologies," IEEE Transactions on Computer-Aided Design of Integrated Circuits and Systems, vol. 25, núm. 1,pp. 58-65, 2006. 
[12] Z. Yu, P. J. Burke, "Microwave Transport in Metallic Single-Walled Carbon Nanotubes," Nano Letters, vol. 5, núm. 7, pp. 1403-1406, Junio, 2005. [en línea]. Disponible en: https://pubs.acs.org/doi/full/10.1021/ nl050738k. Consultado: 5 septiembre, 2018.

[13] M. Sun, Z. Xiao, Y. Chai, Y. Li y P. C. H. Chan, "Inductance Properties of In Situ-Grown Horizontally Aligned Carbon Nanotubes," IEEE Transactions on Electron Devices, vol. 58, núm. 1, pp. 229-235, Enero, 2011. [en línea]. Disponible en: https://ieeexplore.ieee.org/abstract/ document/5628276. Consultado: 10 septiembre, 2018.

[14] I. I. Nefedova, D. V. Lioubtchenko, I. S. Nefedov y A. V. Räisänen, "Conductivity of Carbon Nanotube Layers at Low-Terahertz Frequencies," IEEE Transactions on Terahertz Science and Technology, vol. 6, núm. 6, pp. 840-845, noviembre, 2016. [en línea]. Disponible en: https://ieeexplore.ieee.org/document/7570260. Consultado: 17 septiembre, 2018.

[15] P. Xu, M. C. Hamilton, "Solution-Deposited Carbon Nanotube Flip Chip Interconnect for High-Frequency Applications," IEEE Microwave and Wireless Components Letters, vol. 25, núm. 4, pp. 229-231, Abril, 2015. [en línea]. Disponible en: https://ieeexplore.ieee.org/ document/7045619. Consultado: 19 septiembre, 2018.

[16] H. Li, K. Banerjee, "High-Frequency Analysis of Carbon Nanotube Interconnects and Implications for On-Chip Inductor Design," IEEE Transactions on Electron Devices, vol. 56, núm. 10, pp. 2202-2214, octubre, 2009. [en línea]. Disponible en: https://ieeexplore.ieee.org/ document/5238613. Consultado: 23 Septiembre, 2018.

[17] W-S. Zhao, J. Zheng, Y. Hu, S. Sun, G. Wang, L. Dong, L. Yu, L. Sun, W-Y. Yin, "High-Frequency Analysis of CuCarbon Nanotube Composite Through-Silicon Vias," IEEE Transactions on Nanotechnology, vol. 15, núm. 3, pp. 506-511, mayo, 2016. [en línea]. Disponible en: https:/ /ieeexplore.ieee.org/document/7442879. Consultado: 3 octubre, 2018.

[18] D.A. Frickey, "Conversions Between S, 2, Y, h, ABCD, and T Parameters which are Valid for Complex Source and Load Impedances", IEEE Transactions on Microwave Theory and Techniques, vol. 42, núm. 2, pp. 205-211, febrero, 1994. [en línea]. Disponible en: https://ieeexplore.ieee.org/ document/275248. Consultado: 5 enero, 2020.

[19] A. A. Vyas, C. Zhou, C. Y. Yang, "On-Chip Interconnect Conductor Materials for End-of-Roadmap Technology Nodes," IEEE Transactions on Nanotechnology, vol. 17, núm. 1, pp. 4-10, 2018. [en línea]. Disponible en: https:// ieeexplore.ieee.org/document/7765105. Consultado: 5 diciembre, 2019.

[20] X. Wei, L. Pengfei, Carbon Nanotubes - Synthesis, Characterization, Applications, Siva Yellampalli(Ed.), 2011. [en línea]. Disponible en: https://www.intechopen.com/ books/carbon-nanotubes-synthesis-characterizationapplications/dielectrophoretic-deposition-and-alignmentof-carbon-nanotubes. Consultado: 2 junio, 2019.

[21] L. Jia, Y. Zhang, J. Li, C. You, E. Xie, "Aligned singlewalled carbon nanotubes by Langmuir-Blodgett technique," Journal of Applied Physics, vol. 104, núm. 17, pp. 074318.1-074318.6, agosto, 2008. [en línea]. Disponible en: https://aip.scitation.org/doi/full/10.1063/ 1.2996033. Consultado: 12 junio, 2019.

[22] A. Pacheco-Sánchez, I. Larruz-Castillo, D. Sangani, D. Valdez-Perez, "Comprehensive review on high-frequency CNT-based interconnects", IEEE International Conference on Applied Science and Advanced Technology (iCASAT), Querétaro, Mexico, noviembre 2019. doi: https://dor.org.10.1109/iCASAT48251. 2019.9069515 
